\title{
EDITORIAL
}

\section{Recent Development in Symmetries and Integrability of Difference Equations}

\author{
Xingbiao HU ${ }^{1}$, Qingping $\mathbf{L I U}^{2}$, Senyue $\mathbf{L O U}^{3}$, Changzheng $\mathbf{Q U}^{4}$, \\ Youjin ZHANG ${ }^{5}$ \\ 1 Institute of Computational Mathematics and Scientific Engineering Computing, Academy \\ of Mathematics and Systems Science, Chinese Academy of Sciences, Beijing 100190, China \\ 2 Department of Mathematics, China University of Mining and Technology, Beijing 100083, \\ China \\ 3 Shanghai Key Laboratory of Trustworthy Computing, East China Normal University, \\ Shanghai 200062, China; Faculty of Sciences, Ningbo University, Ningbo 315211, China \\ 4 Center for Nonlinear Studies, Ningbo University, Ningbo 315211, China \\ 5 Department of Mathematical Sciences, Tsinghua University, Beijing 100084, China
}

(C) Higher Education Press and Springer-Verlag Berlin Heidelberg 2013

Difference equations or discrete systems are mathematical models of various fields such as physics, chemistry, biology, and economics and have been subjects of extensive study of both pure mathematicians and applied mathematicians. Through its interaction with modern integrable systems, the theory of difference equations is enriched greatly and has been undergoing a rapid development.

SIDE-10, the tenth of a series of biennial conferences devoted to Symmetries and Integrability of Difference Equations and related topics, was held during 10-16 June, 2012 at Ningbo, China. It was sponsored and supported by the National Natural Science Foundation of China, Ningbo Association of Science and Technology, Ningbo University, Academy of Mathematics and Systems Science of Chinese Academy of Sciences, China University of Mining and Technology (Beijing), Tsinghua University, and Shanghai University. The conference attracted over 100 participants from more than a dozen of countries. During the conference, 44 contributed talks were arranged and the topics covered by the meeting include

- Difference algebra and integrable maps

- Discrete differential geometry and integrable lattices

- Discrete Painlevé equations and Garnier systems and nonlinear special functions

- Numerical algorithms and integrability

\footnotetext{
Received August 6, 2013

E-mail: hxb@lsec.cc.ac.cn, qpl@cumtb.edu.cn, lousenyue@nbu.edu.cn, quchangzheng@nbu.edu.cn,youjin@mail.tsinghua.edu.cn
} 
- Orthogonal polynomials and discrete complex function theory

- Quantum discrete systems and integrability

- Spectral theory and solution methods

- Symmetries and conservation laws

On the suggestions of the SIDE steering committee, Jarmo Hietarinta, Decio Levi, and Frank Nijhoff in particular, we have compiled this special issue of Frontiers of Mathematics in China. It is the wish of both SIDE steering committee and the guest editors that the present collection of papers, although covering only a small portion of the talks presented at the conference, may reflect the recent development and findings in this active field. All contributions in this issue were peer reviewed under the standard of the journal. We would like to thank all authors for their contributions to this issue, and all referees for providing timely and constructive reviews. We would also like to thank Mr. Shannian Lu, the managing editor of Frontiers of Mathematics in China for his enthusiastic support while preparing this issue. 